\section{Front-to-back ratio improvement of printed antennas based on electrically small resonators for microwave presence detectors}

\section{P. Aguilà, S. Zuffanelli, G. Zamora, F. Paredes, F. Martín} and J. Bonache

The improvement of the front-to-back ratio (FBR) in low-profile antennas to be used in presence detection devices is explored in this work. The main idea is to use electrically small resonators as radiating elements. This minimizes the electric currents in the boundary of the ground plane at the working frequency, thus reducing the backward radiation. The choice of the resonant particle and its electromagnetic properties, along with the antenna structure, are discussed. The simulated results indicate that there is good impedance matching $(-18 \mathrm{~dB})$ at the operating frequency $(3 \mathrm{GHz})$ and an excellent FBR of $24 \mathrm{~dB}$. These characteristics are validated experimentally and the FBR is compared to that of a conventional patch antenna.

Introduction: Most modern buildings incorporate automatic presence detectors for security, energy saving and domotics. These devices usually integrate infrared or ultrasonic sensors and hence they are unable to detect objects through walls, floors or ceilings. This fact restricts the operational region of the detector to the room where it is placed. To overcome this difficulty, presence detectors operating at microwaves have been proposed. These devices use a microwave signal transmitted by an antenna, which is reflected back by the target to be detected. Since the functionality of these microwave detectors can be extended over several meters, it is important to restrict the sensing region to avoid undesired readings. The most important parameter that quantifies this problem is the front-to-back ratio (FBR) of the emitter antenna, defined as the ratio of the power sent in the direction of detection over the power sent in the opposite direction. Thus, the FBR must be maximized for an adequate performance.

Low cost microwave detectors usually incorporate common planar microstrip antennas, consisting of a rectangular metal patch placed on top of a dielectric substrate mounted over a large ground plane. This type of antennas is supposed to provide a radiation diagram located in the half-space due to the presence of the ground plane. Nevertheless, since this kind of antennas can be viewed as an open circuited transmission line, the electric field at the edges of the conductor patch will spread into the surrounding substrate. This results in the extension of currents over a significant area of the ground plane. For this reason, the ground plane should be maintained electrically large, in order to preserve the radiation diagram and hence minimizing the radiation to the back side of the antenna. However, in some applications, the size of the antenna is a critical issue and should be minimized. To overcome this drawback, we propose the use of an electrically small resonator, which concentrates the currents around its geometry, as a radiator. Considering that the particle is relatively close to the ground plane (and also its image, located on the opposite side of the ground plane [1]), the induced currents in the ground plane are expected to be concentrated in a relative small region around the resonator. This fact leads to a reduction of the currents at the edges of the ground plane, resulting in the optimization of the FBR when its dimensions are decreased.
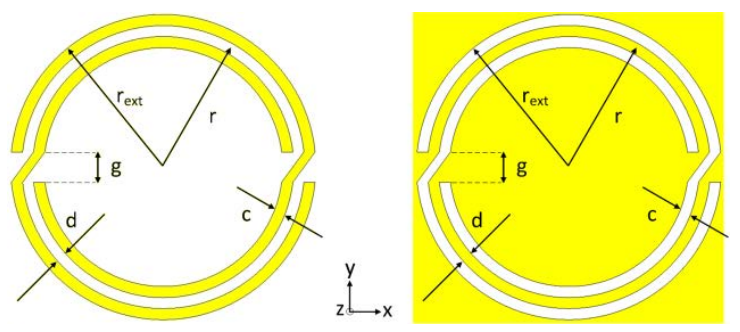

Fig. 1 Non-bianisotropic split ring resonator (left) and its dual counterpart (right). Metal is depicted in yellow, slot in white.
Split ring resonators (SRRs) and complementary split ring resonators (CSRRs) have been widely used to obtain enhanced functionality in conventional patch antennas [2]. However, the use of these resonators as radiating elements has not been explored so much. In this work, the functionality of these resonant elements as radiators in antennas with enhanced FBR is studied.

Antenna design: Let us first analyse the polarizabilities of the previous particles (SRRs and CSRRs). This parameter indicates the response (polarization) of the particle to the exciting field. To avoid undesired cross-polarization effects, we will indeed focus on the nonbianisotropic particles, i.e. the non-bianisotropic split ring resonator (NB-SRR) and its complementary counterpart, the non-bianisotropic complementary split ring resonator (NB-CSRR) (Fig. 1). According to [3], the NB-SRR exhibits an axial magnetic dipole moment at the fundamental resonance, and hence the particle can be excited by a magnetic field parallel oriented to its axis (z-direction in Fig. 1) at that frequency. Conversely, the NB-SRR exhibits an electric dipole moment in the $y$-direction at the second resonance frequency. Note that this particle cannot be excited by an axially incident electromagnetic wave provided the resonator and the ground plane are parallel oriented (the most convenient implementation from the fabrication point of view) and closely spaced. This is due to the boundary conditions forced by the presence of the ground plane (which cancels the tangential electric field) and the absence of axial magnetic field associated to this particular wave incidence.

For the NB-CSRR, an electric dipole moment is generated at the fundamental resonance if the particle is excited by an axial electric field (z-direction in Fig. 1). At the second resonance, this particle exhibits a magnetic dipole moment in the $y$-direction [3,4]. Thus, since a tangential component of the magnetic field is present for an axially incident electromagnetic wave (and do not vanishes near the ground plane), a NB-CSRR working at the second resonance is used for the present application.

Simulated results and measurements: Fig. 2 shows the geometry of a NB-CSRR (modelled in CST-Microwave Studio) designed to exhibit its second resonance at $3 \mathrm{GHz}$ (S-band). A conductor strip is placed on the back side of the substrate where the resonator is etched in order to provide an external feed to the particle. The length of this metallic strip is set to roughly $\lambda / 2$ at the second resonance. Hence, the maximum current density occurs at the midpoint of the line, resulting in a strong magnetic coupling between the particle and the strip. With this length and width (indicated below) good impedance matching with the $50 \Omega$ input port is achieved. The dimensions of the metal plate where the particle is etched are $40 \times 40 \mathrm{~mm}^{2}$. The substrate is Rogers RO3010 with relative permittivity $\varepsilon_{r}=10.2$ and thickness $h=1.27 \mathrm{~mm}$. The values of the geometrical parameters of the NB-CSRR are $c=0.44 \mathrm{~mm}$, $r_{\text {ext }}=6.06 \mathrm{~mm}(\approx \lambda / 17), r=5.40 \mathrm{~mm}, d=0.44 \mathrm{~mm}$ and $g=1.2 \mathrm{~mm}$. For the transmission line, $L=38.73 \mathrm{~mm}$ and $W=4.1 \mathrm{~mm}$.

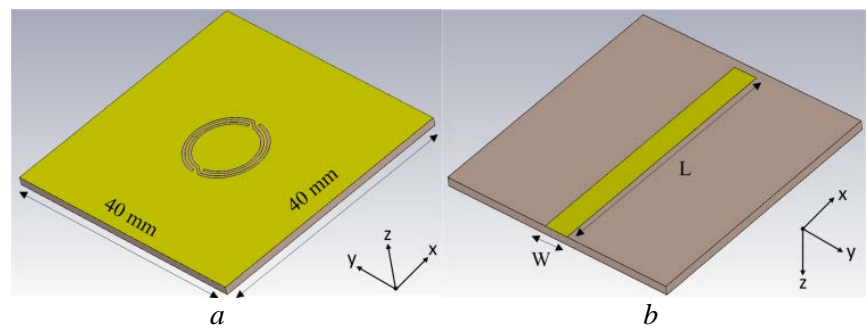

Fig. 2 Simulation model of a NB-CSRR excited with a microstrip line $a$ Front side of the configuration $b$ Back side of the configuration

The structure of the whole antenna and the manufactured prototype device are shown in Fig. 3. The dimensions of the ground plane are 100 $\times 100 \mathrm{~mm}^{2}$, and it has been spaced out from the NB-CSRR metal layer in order to obtain a reasonable operational bandwidth. This spacing has been modelled in simulation as an air layer, and implemented through a piece of foam of thickness $T=8.73 \mathrm{~mm}$. 


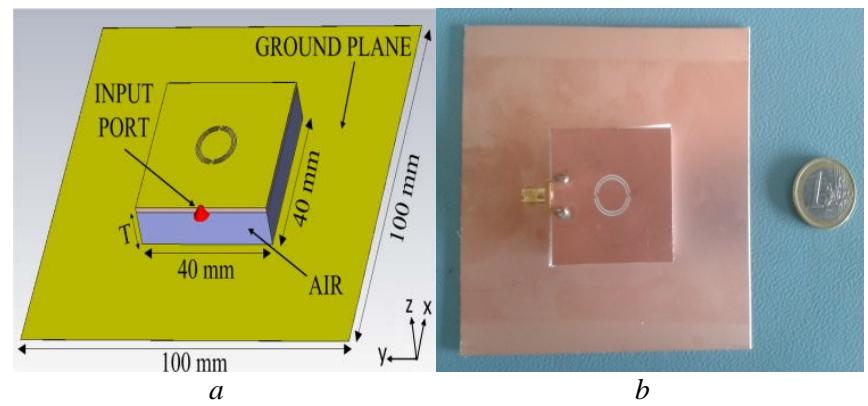

Fig. 3 Geometry of the designed antenna

$a$ Simulation model view

$b$ Manufactured antenna prototype

A comparison between the simulated (obtained by means of CSTMicrowave Studio) and measured (obtained by means of the Agilent E8364B network analyser) reflection coefficient is depicted in Fig. 4. The matching in the vicinity of $3 \mathrm{GHz}$ is better than $-15 \mathrm{~dB}$. The CSTpredicted current distribution of the antenna, shown in Fig. $5 a$, verifies that the current concentrates near the resonator. Fig. $5 b$ depicts the simulated radiation pattern exhibited by the structure.

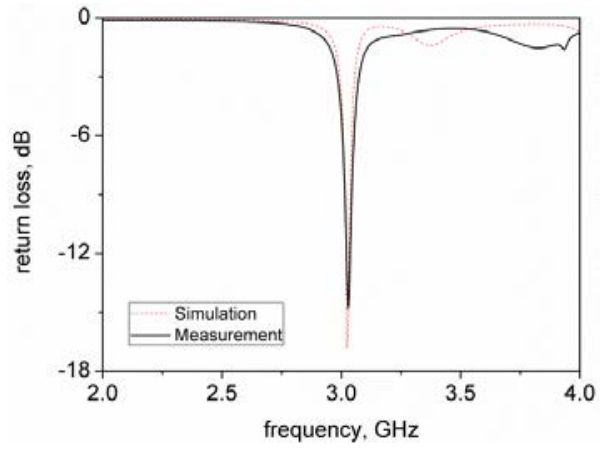

Fig. 4 Comparison of measured and simulated power reflection coefficient

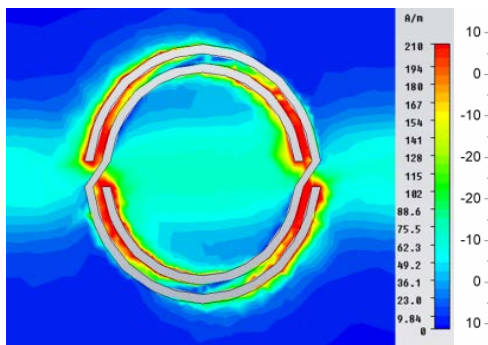

$a$

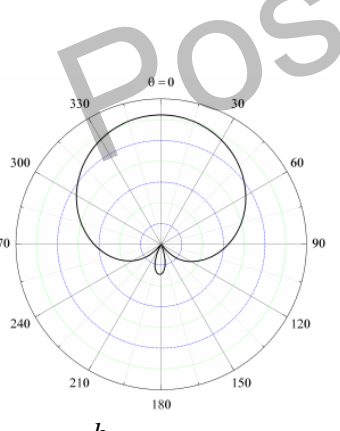

$b$
Fig. 5 Antenna characteristics simulated with CST Microwave Studio $a$ Current distribution of the antenna, XY-plane $b$ Radiation pattern (gain) of the antenna, YZ-plane

The FBR is obtained as the difference in $\mathrm{dB}$ of the gain in the direction of maximum gain and the gain in the opposite direction $\left(180^{\circ}\right.$ from that specified maximum). According to Fig. $5 b$, for $\theta=0^{\circ}$ the gain is $6 \mathrm{~dB}$, and for $\theta=180^{\circ}$ it is $-18 \mathrm{~dB}$, so the simulated front-to-back ratio is $\mathrm{FBR}=24 \mathrm{~dB}$. To experimentally validate the FBR, the antenna is placed in a TEM cell and is excited through a vector signal generator (Agilent N5182A) with a pure tone of incident power equal to $0 \mathrm{dBm}$ at $3 \mathrm{GHz}$. The antenna response is finally displayed in the vector signal analyser (Agilent N9020A). The received power is $-29.9 \mathrm{dBm}$ when the signal is received from the front side of the antenna and $-53.8 \mathrm{dBm}$ when the antenna is rotated $180^{\circ}$. Thus, the measured front-to-back ratio is $\mathrm{FBR}=23.9 \mathrm{~dB}$, very close to its simulated value. The structure of a conventional patch antenna designed at the same operating frequency $(3 \mathrm{GHz})$ is shown in Fig. $6 a$. Fig. $6 b$ shows its simulated radiation pattern obtained by CST Microwave Studio. The gain is $8.8 \mathrm{~dB}$ (maximum value) for $\theta=0^{\circ}$ and $-6.2 \mathrm{~dB}$ for $\theta=180^{\circ}$, so the simulated front-to-back ratio is FBR $=15 \mathrm{~dB}$. Thus, by using a small resonator as the main radiator (a NB-CSRR), the FBR can be improved $9 \mathrm{~dB}$.
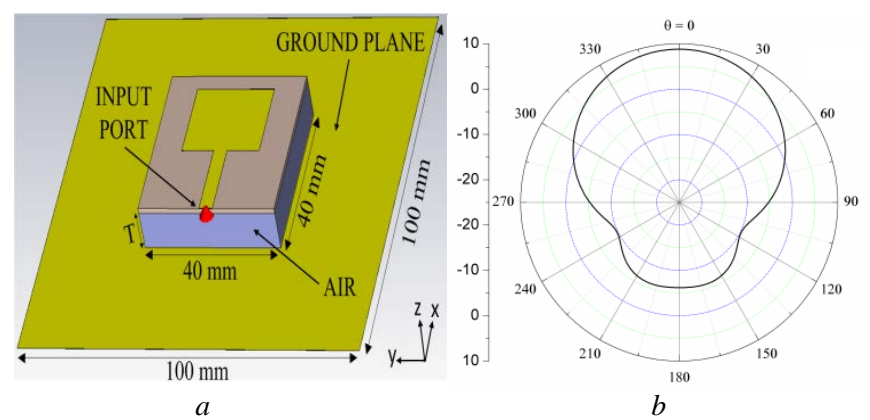

Fig. 6 Geometry of the microstrip antenne $a$ Simulation model view

$b$ Radiation pattern (gain) of the antenna, YZ-plane

From the radar equation [1], it can be seen that, for a given threshold detection power, the maximum detection distance is proportional to the square root of the antenna gain. Thus, the ratio between the maximum distances of detection for objects placed in the front and in the back sides of the detector can be calculated as:

$$
R_{\text {Front }} / R_{\text {Back }}=\sqrt{F B R}
$$

The presented NB-CSRR-based antenna shows a ratio $R_{\text {Front }} / R_{\text {Back }}=$ 15.7, while the patch antenna presents a ratio of 5.6. This represents a significant improvement for the case of the NB-CSRR-based antenna. Table 1 shows a performance comparison for both types of antennas.

Table 1: Performance comparison of both antennas

\begin{tabular}{|c|c|c|c|c|}
\hline \multirow{2}{*}{$\begin{array}{c}\text { NB-CSRR antenna } \\
\text { (measured) }\end{array}$} & $\operatorname{Pr}_{\text {front }}(\mathrm{dBm})$ & $\mathbf{P r}_{\text {back }}(\mathrm{dBm})$ & $\mathbf{F B R}(\mathrm{dB})$ & $\mathbf{R}_{\text {Front }} / \mathbf{R}_{\text {Back }}$ \\
\hline $\begin{array}{c}\text { Patch antenna } \\
\text { (simulated) }\end{array}$ & -29.9 & -53.8 & 23.9 & 15.7 \\
\hline
\end{tabular}

Conclusion: The FBR improvement in printed antennas has been studied in this work. Due to the particle polarizabilities, a NB-CSRR working at the second resonance has been chosen as radiating element. The geometrical parameters of the antenna have been adjusted to a working frequency of $3 \mathrm{GHz}$. Its model has been electromagnetically simulated with CST Microwave Studio, showing a good impedance matching of $-18 \mathrm{~dB}$ and a very high front-to-back ratio of FBR $=24 \mathrm{~dB}$. This performance has been confirmed experimentally, and the FBR of the proposed antenna has been compared to that of a microstrip patch antenna, showing an enhancement of $9 \mathrm{~dB}$. The ratio $R_{\text {Front }} / R_{\text {Back }}$ has been increased roughly by a factor 3 , representing a significant performance improvement of the NB-CSRR-based antenna as compared to the patch antenna.

P. Aguilà, S. Zuffanelli, G. Zamora, F. Paredes, F. Martín and J. Bonache (CIMITEC, Departament d'Enginyeria Electrònica, Universitat Autònoma de Barcelona, 08193 Bellaterra, Spain).

E-mail: pau.aguila@uab.cat

\section{References}

1. Balanis, C.A.: ‘Antenna Theory: Analysis and Design' (John Wiley \& Sons, Inc., Hoboken, New Jersey, 2005, third edition)

2. Paredes, F. et al.: 'Free-space and on-metal dual-band tag for UHFRFID applications in Europe and USA', Progress In Electromagnetics Research, vol. 141, 577-590, 2013

3. García-García, J. et al.: 'On the resonances and polarizabilities of Split ring resonators', J. Appl. Phys. 98, 33103 (2005).

4. Falcone, F. et al.: 'Babinet Principle Applied to the Design of Metasurfaces and Metamaterials', Phys. Rev. Lett. vol. 93, 197401, Nov. 2004. 